\title{
A Simple and Efficient Procedure for Identifying the Compressing Stage in Two-Stage Amplifiers
}

\author{
Anton N. Atanasov"\#1, Waqam H. R. A. Mukhtar Ahmad \#, Mark S. Oude Alink", Frank E. van Vliet"\# \\ \#Integrated Circuit Design, University of Twente, Enschede, Netherlands \\ *Defence, Safety \& Security, TNO, The Hague, Netherlands \\ 1a.n.atanasov@utwente.nl
}

\begin{abstract}
We propose a fast and simple method to accurately determine the compressing stage in a two-stage amplifier system based on reverse intermodulation and hot S-parameter measurements. The method uses no specialized hardware and needs little reconfiguration, simplifying the measurements. We demonstrate its validity over a broad range of amplifiers designed in various semiconductor technologies. This method is a very useful tool in understanding or debugging amplifier designs.

Keywords - compression, intermodulation, reverse intermodulation, power amplifiers, nonlinearity
\end{abstract}

\section{INTRODUCTION}

Power amplifiers (PAs) for modern telecommunications and radar are often highly integrated multistage devices, which make them increasingly complex to design and debug. For example, a PA will not be able to deliver the designed power if the first stage compresses too early. Designers have to rely on limited measurements and their own experience to understand the root cause of such early compression in a PA or even just identifying the limiting stage, resulting in several costly design iterations before design tolerances are met [1]. Currently available debugging techniques either rely on sophisticated measurement equipment [2], [3], difficult time-domain measurements [4], idealized analysis [5], or placing measurement probes between the active stages, if possible at all.

In this paper we present a simple method for identifying the compressing stage in two-stage amplifiers based on an empirical relation used to estimate reverse intermodulation distortion (RIMD) from intermodulation distortion (IMD) measurements [6]. The proposed method is similar in nature and complexity to measuring hot S-parameters [7] and offers good insights into the behaviour of a two-stage PA while being simple and easy to implement without requiring expensive nonlinear VNAs.

In Section II we demonstrate the generality of our empirical RIMD estimator by providing a mathematical relation between IMD and RIMD using a general large-signal transistor model [8]. We show that the error between the RIMD estimator and measurement result can be used to determine the compressing stage in a two-stage PA. In Section III a complete measurement procedure is presented, including representative measurements of PAs implemented in several semiconductor technologies to verify the validity of the procedure. Finally, the conclusions are summarized in Section IV.

\section{Stage COMPRESSION IDENTIFICATION USING RIMD}

\section{A. RIMD Estimator Analysis}

In our previous work [6] we examined the reverse intermodulation products generated when a PA is transmitting power at one frequency while reverse power, at a close frequency offset, is injected into its output. We showed how they relate to regular IMD products generated using the same power levels. We defined a simple $\mathrm{dB}$-scale relation to estimate the strongest RIMD component, $\overline{\mathrm{RIM} 31}$, from the measurement of the strongest IMD component, IM31, via general device parameters

$$
\overline{\mathrm{RIM} 31} \triangleq 20 \log _{10}\left(\left|\Gamma_{\text {out }}\right|\right)-G_{\mathrm{A}}+\mathrm{IM} 31,
$$

where $20 \log _{10}\left(\left|\Gamma_{\text {out }}\right|\right)$ is the active output reflection coefficient and $G_{\mathrm{A}}$ is the available gain. The relationship states that IM31 interacts with $G_{\mathrm{A}}$ in the same way as RIM31 interacts with $20 \log _{10}\left(\left|\Gamma_{\text {out }}\right|\right)$. The estimator error was measured to be approximately $\pm 3.5 \mathrm{~dB}$ over several frequency offsets, which is reasonable when relating behaviours $30 \mathrm{~dB}$ apart.

To demonstrate the general weakly-nonlinear similarity between both processes we consider the large-signal Angelov model [8], a representative model for multiple III-V device technologies, and are, in this context, only interested in its non-linear properties. We express the normalized drain current as

$$
I_{\mathrm{ds}}=\left(1+\tanh \left(V_{\mathrm{gs}}\right)\right) \tanh \left(\alpha V_{\mathrm{ds}}\right)\left(1+\lambda V_{\mathrm{ds}}\right),
$$

where $V_{\mathrm{gs}}$ is the gate-source voltage, $V_{\mathrm{ds}}$ is the drain-source voltage, $\lambda$ is the channel length modulation parameter and $\alpha$ is the saturation voltage parameter.

We first consider the IMD case, in which the stimulus becomes $V_{1}+V_{2}$, where $V_{i}$ are two signals at different frequencies. In this configuration $\tanh \left(\alpha V_{\mathrm{ds}}\right)\left(1+\lambda V_{\mathrm{ds}}\right)=\mathrm{A}$ can be treated as a constant and thus, without loss of generality, we have

$$
I_{\mathrm{ds}} \approx \mathrm{A}\left(1+\tanh \left(V_{1}+V_{2}\right)\right)=\mathrm{A} \frac{2 e^{2\left(V_{1}+V_{2}\right)}}{e^{2\left(V_{1}+V_{2}\right)}+1} .
$$

Next we consider the RIMD case, where the now reverse-injected signal, $V_{2}$, propagates, due to the reciprocity of the output-matching network, towards the drain-terminal where it causes a variation on the drain-source voltage, $V_{\mathrm{ds}}$, 
which influences $I_{\mathrm{ds}}$. The result can be simplified by setting $1+\lambda\left(V_{\mathrm{ds}}+V_{2}\right)=\mathrm{B}\left(V_{2}\right)$ such that

$$
I_{\mathrm{ds}} \approx \mathrm{B}\left(V_{2}\right) \frac{2 e^{2\left(V_{1}+\alpha\left(V_{\mathrm{ds}}+V_{2}\right)\right)}-2 e^{2 V_{1}}}{e^{2\left(V_{1}+\alpha\left(V_{\mathrm{ds}}+V_{2}\right)\right)}+e^{2 V_{1}}+e^{2\left(\alpha\left(V_{\mathrm{ds}}+V_{2}\right)\right)}+1} .
$$

Equations 3 and 4 both have similar two-tone exponential terms that result in similar intermodulation products. The most observable difference between them is that (4) has additional single tone terms in both the numerator and denominator. This causes the generated RIMD terms from the output to be attenuated. This is to be expected, as the reverse power does not experience any gain when generating RIMD, while also being attenuated by the output reflection coefficient.

\section{B. Identification Methodology}

The RIMD estimator can be used to determine which stage enters compression first because it will agree with measurements, within some margin, only when the dominant compression occurs in the last stage of the PA. This is because if the IMD and RIMD products are created at the same stage, they can be related to one another via Eqn. (1).

If the dominant compression of the PA occurs at the first stage, the IMD products will increase substantially, due to $G_{\mathrm{A}}$ of the whole PA, while the RIMD ones will not. The second stage will not be able to generate such strong intermodulation products by itself. This divergence between IMD and RIMD power levels serves as the indicator. The compression point of the first stage will limit the entire PA and the RIMD measurements will differ from the estimate by some amount, $\Delta_{e}$, which we define as

$$
\Delta_{e} \triangleq|\overline{\operatorname{RIM} 31}-\mathrm{RIM} 31| \text {. }
$$

The error is caused by the increased IMD products together with the available gain of the second stage only. If this gain is not known precisely then the magnitude provides no insight. If both stages are linear, then the IMD and RIMD products will require high dynamic range to measure accurately.

On the other hand, if both stages are driven in strong compression, the IMD components of the first stage will be indistinguishable from those generated by the second stage and $\Delta_{e}$ will be small, providing no insight. Thus, the PA must be driven such that it operates under-large signal conditions, but not in strong compression, similar to the $\mathrm{P} 1 \mathrm{~dB}$ compression point as is done with hot S-parameter measurements [9].

As such, the procedure involves first measuring the available gain, $G_{\mathrm{A}}$, of the device. Next, while the device operates at its P1dB compression point, the IM31, RIM31 and $\Gamma_{\text {out }}$ are measured so that $\Delta_{e}$ can be computed.

\section{COMPRESSING STAGE IDENTIFICATION VERIFICATION}

To validate the utility of the RIMD estimator we use a set of different HBT [10]-[13] and one E-PHEMPT [14] single-stage PAs to construct several two-stage device under test (DUT) combinations. The biasing of either stage is adjusted so as to change which stage of the DUT enters dominant compression, allowing us to emulate designs that are compressing in either the first or the second stage. The RIMD measurements are compared to the estimates and the magnitude of the error is used to identify the compressing stage.

\section{A. Measurement Setup}

The first step in identifying the DUT's compressing stage is characterizing $G_{\mathrm{A}}$. The available gain is measured with a single tone input power sweep at the frequency of interest. The IMD behaviour of the DUT is measured with the setup shown in Figure 1a). A main tone, $P_{1}$, and a probe tone, $P_{2}$, are fed at the input of the DUT. The probe tone is chosen such that it is $10 \mathrm{~dB}$ weaker than $P_{1}$, so that it does not influence the large-signal behaviour, in line with regular hot $\mathrm{S}$-parameter measurements [7]. The lower $P_{2}$ is, the lower its influence will be on the DUT, however the lower IMD and RIMD products will require increased dynamic range from the measurement equipment. The main tone is set to the $\mathrm{P} 0.1 \mathrm{~dB}, \mathrm{P} 1 \mathrm{~dB}$ and $\mathrm{P} 3 \mathrm{~dB}$ compression points of the DUT and is offset by frequency $\Delta_{f}$ of $[1,10,100,150] \mathrm{MHz}$ from the probe tone. The frequency offsets are chosen such that they cover a representative range, while still close enough to have the same S-parameters.The strongest IMD product, IM31, is observed and recorded using a spectrum analyzer via a $20 \mathrm{~dB}$ directional coupler.

Measurement of the RIMD behavior is done using the setup shown in Figure 1b). The $P_{2}$ probe tone is now fed at the output of the DUT and isolated through a circulator. First, the active $\left|\Gamma_{\text {out }}\right|^{2}$ is measured for the given $\Delta_{f}$ and compression point. Both tones, $P_{1}$ and $P_{2}$, have the same amplitudes as with the IMD measurement. The RIMD products, which are much weaker than their IMD counterparts, are also observed with the spectrum analyzer via the same coupler.

For accuracy concerns, special care was taken to have only a minimal amount of reconfiguration between IM31 and RIM31 measurements, such that only two devices needed to be swapped. The fact that no specialized hardware is required facilitates a wide adoption of the method.

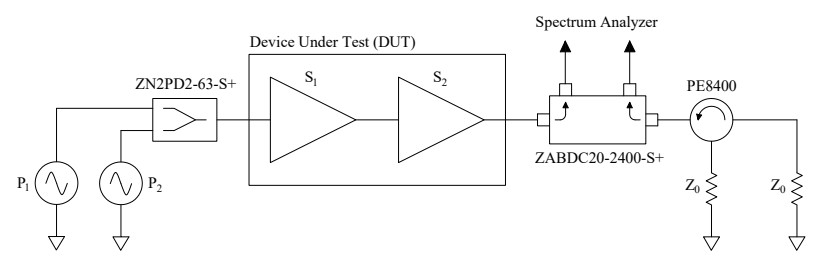

(a) IMD measurement setup

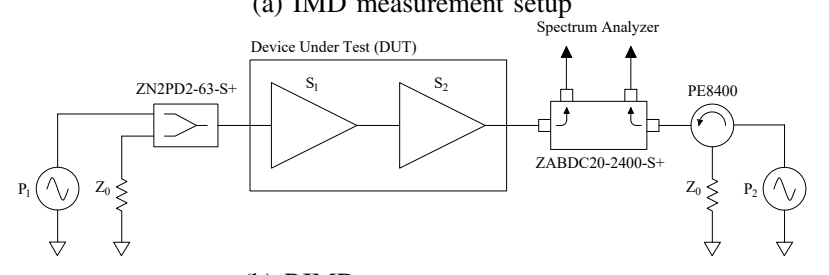

(b) RIMD measurement setup

Fig. 1. RIMD and IMD measurement setups. In both cases the generator tones are kept the same. 


\section{B. Measurement Results}

Figure 2a) shows the output spectrum, after the $20 \mathrm{~dB}$ coupler, of an IMD measurement of a DUT consisting of ZX60-V82-S+ (HBT) input stage and ZX60-P33ULN+ (E-PHEMPT) output stage. The DUT is biased such that the second stage creates dominant compression. The input powers are $P_{1}=2 \mathrm{dBm}$ at $1.51 \mathrm{GHz}$ and $P_{2}=-12 \mathrm{dBm}$ at $1.50 \mathrm{GHz}\left(\Delta_{f}=10 \mathrm{MHz}\right)$, and the output powers are $26.8 \mathrm{dBm}$ and $12.8 \mathrm{dBm}$, respectively. The DUT operates at its $P_{1 \mathrm{~dB}}$ compression point, with $G_{\mathrm{A}}=24.8 \mathrm{~dB}$. The difference in input power levels results in the IM3 components having different magnitudes and slopes, the strongest one being IM31 at $1.52 \mathrm{GHz}$ with a slope of $1 \mathrm{~dB} / \mathrm{dB}$, while the second intermodulation component, IM 32 , is at $1.49 \mathrm{GHz}$ and has a slope of $2 \mathrm{~dB} / \mathrm{dB}$ and is weaker in magnitude.

Figure 2b) shows the output spectrum of an RIMD measurement on the same DUT. Both $P_{1}$ and $P_{2}$ remain unchanged in power and frequency, but $P_{2}$ is applied to the output of the DUT. The measured output power is $P_{\mathrm{O} 1}=$ $-3.2 \mathrm{dBm}$ and $P_{\mathrm{O} 2}=-63.0 \mathrm{dBm}$, which tells us (Eqn. (1)) that the active $\left|\Gamma_{\text {out }}\right|^{2}=-8.3 \mathrm{~dB}$. As with the IMD measurement, the strongest reverse intermodulation component, RIM31, located at $1.52 \mathrm{GHz}$, has a slope of $1 \mathrm{~dB} / \mathrm{dB}$, while the second reverse intermodulation component, RIM32, has a slope of $2 \mathrm{~dB} / \mathrm{dB}$.

The RIMD estimator is in good agreement with measurements, resulting in $\Delta_{e}=|34.3-36.4|=2.1 \mathrm{~dB}$. The rest of the frequency offsets yield $1.7 \mathrm{~dB}$ at $1 \mathrm{MHz}, 3.0 \mathrm{~dB}$ at $100 \mathrm{MHz}$ and $1.1 \mathrm{~dB}$ at $150 \mathrm{MHz}$, respectively. When the same DUT is biased such that the first stage enters compression first, and the IMD and RIMD measurements are repeated under the exact same conditions, the estimator error becomes $13.5 \mathrm{~dB}$. Figure 3 shows the new IMD and RIMD results. The other frequency offsets yield an estimator error of $14.8 \mathrm{~dB}$ at $1 \mathrm{MHz}, 16.5 \mathrm{~dB}$ at $100 \mathrm{MHz}$ and $16.5 \mathrm{~dB}$ at $150 \mathrm{MHz}$. Table 1 shows the RIMD estimator error, $\Delta_{e}$, for all $\Delta_{f}$ offsets for several DUT combinations which have been biased such that either the first stage or the second stage enter compression first. As pointed out earlier, the most consistent results are obtained when $P_{1}$ is set around the $\mathrm{P} 1 \mathrm{~dB}$ compression point. Significantly lower values (e.g. P0.1dB) lead to measurement inaccuracies due to the low signal levels, significantly higher values (e.g. P3dB) removes the dominance of the nonlinearity, and hence obfuscates the results. Additionally, the most distinct performance is obtained when $\Delta_{f}=[1,10] \mathrm{MHz}$. At higher frequency offsets there seems to be no guarantee the device parameters will remain the same.

The method is able to identify the compressing stage most consistently at small $\Delta_{f}$, such as $1 \mathrm{MHz}$ and $10 \mathrm{MHz}$, when the DUT operates around the P1dB compression point. At higher frequency offsets such as $100 \mathrm{MHz}$ and $150 \mathrm{MHz}$ the estimator cannot be trusted consistently. It can be concluded that when $\Delta_{e}<4 \mathrm{~dB}$ the second stage enters compression first. When $\Delta_{e}>8 \mathrm{~dB}$, a significant difference, the first stage enters compression first, limiting the compression point of the entire

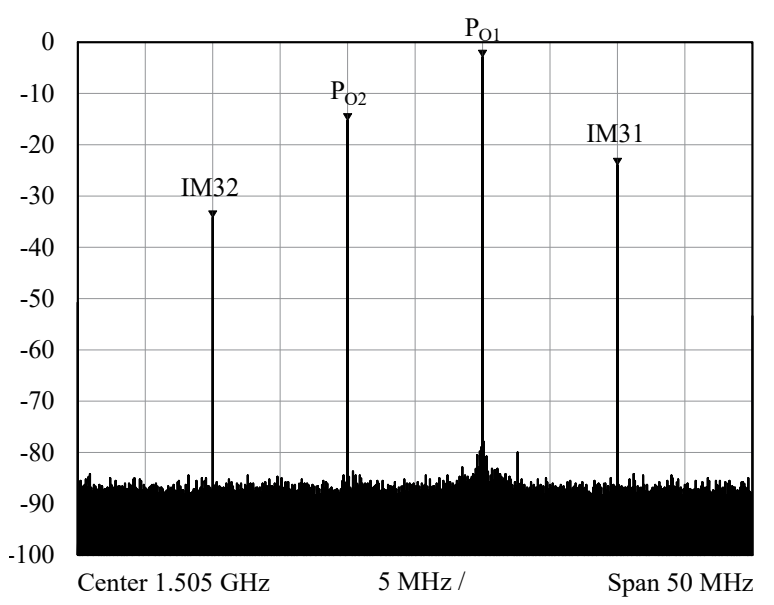

(a) IMD spectrum with $P_{\mathrm{O} 1}=-3.1 \mathrm{dBm}, P_{\mathrm{O} 2}=-15.5 \mathrm{dBm}$, $\mathrm{IM} 31=-29.2 \mathrm{dBm}$ and $\mathrm{IM} 32=-34.3 \mathrm{dBm}$.

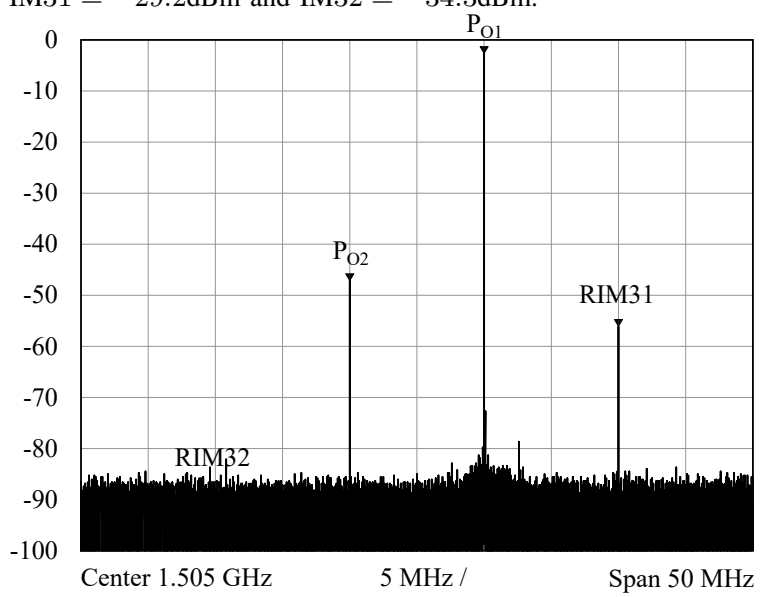

(b) RIMD spectrum with $P_{\mathrm{O} 1}=-7.3 \mathrm{dBm}, P_{\mathrm{O} 2}=-33.7 \mathrm{dBm}$, RIM31 $=-62.3 \mathrm{dBm}$ and RIM32 $=-82.8 \mathrm{dBm}$

Fig. 2. IMD and RIMD spectra, via the $20 \mathrm{~dB}$ coupler, of DUT consisting of ZX60-V82-S+ (HBT) input stage and ZX60-P33ULN+ (E-PHEMPT) output stage with $\Delta_{f}=10 \mathrm{MHz}, P_{1}=2 \mathrm{dBm}$ and $P_{2}=-12 \mathrm{dBm}$. The DUT compresses at the output stage.

system.

\section{CONCLUSION}

In this work we have further explored the relationship between IMD and RIMD and applied the RIMD estimator proposed in [6] to identify which stage enters compression first in a two-stage PA device using only linear measurement equipment. To validate our assumptions we measured seven two-stage DUTs consisting of combinations E-PHEMPT and HBT-based single-stage PAs. The different technologies and topologies of each device support the general applicability of the estimator.

The DUT configurations were evaluated at several frequency offsets and compression points. At around the P1dB compression point and $\Delta_{f}=[1,10] \mathrm{MHz}$ the estimator error, $\Delta_{e}$, is able to identify the compressing stage consistently. The well-designed DUT configurations resulted in $\Delta_{e}<4 \mathrm{~dB}$, while the DUTs which compressed at the first stage had $\Delta_{e}>8 \mathrm{~dB}$. 


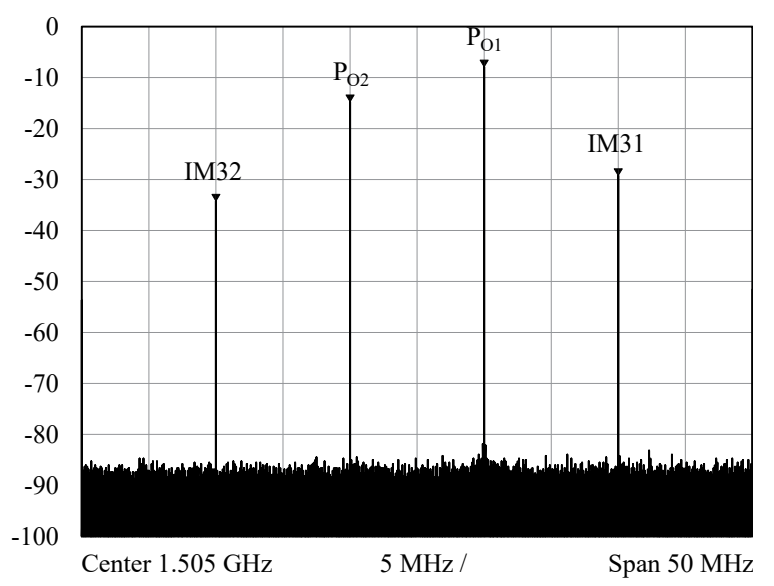

(a) IMD spectrum with $P_{1}=-8.0 \mathrm{dBm}, P_{2}=-14.9 \mathrm{dBm}$, $\mathrm{IM} 31=-29.2 \mathrm{dBm}$ and IM32 $=-34.3 \mathrm{dBm}$.

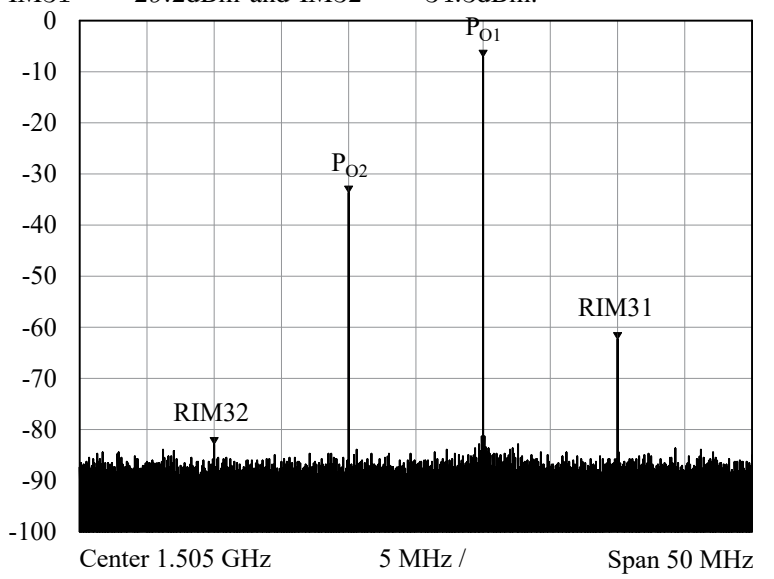

(b) RIMD spectrum with $P_{1}=-7.3 \mathrm{dBm}, P_{2}=-33.7 \mathrm{dBm}$, RIM31 $=-62.3 \mathrm{dBm}$ and RIM $32=-82.8 \mathrm{dBm}$.

Fig. 3. IMD and RIMD spectra, via the $20 \mathrm{~dB}$ coupler, of DUT consisting of ZX60-V82-S+ (HBT) input stage and ZX60-P33ULN+ (E-PHEMPT) output stage with $\Delta_{f}=10 \mathrm{MHz}, P_{1}=2 \mathrm{dBm}$ and $P_{2}=-12 \mathrm{dBm}$. The DUT compresses at the input stage.

\section{ACKNOWLEDGMENT}

We would like to acknowledge Dr. Diogo Ribeiro from TNO for his help with equipment and measurements.

\section{REFERENCES}

[1] C. Tsironis, B. Li, D. Dubouil, and A. Henin, "A new method to test and design multistage power amplifiers using load-pull data," in 1999 29th European Microwave Conference, vol. 2, 1999, pp. 228-231.

[2] D. E. Root, J. Verspecht, and J. Horn, X-Parameters. Cambridge University Press, 2017.

[3] P. Ryan, K. Davis, and S. Rawat, "A case study of two-stage fault location," in 30th Annual Proceedings Reliability Physics 1992, 1992, pp. 332-337.

[4] J. Martens, "Non-linear effects and the use of network analyzer time domain," in 2009 73rd ARFTG Microwave Measurement Conference, 2009, pp. 1-5.

[5] J.-S. Ko, H.-S. Kim, B. Kim, and B.-H. Park, "High-frequency intermodulation analysis of cascode amplifier," in Proceedings of Second IEEE Asia Pacific Conference on ASICs. AP-ASIC 2000 (Cat. No.00EX434), 2000, pp. 167-170.
Table 1. Estimator error, $\Delta_{e}$, when compression occurs in the output stage (2) or the input stage (1) of the DUT at P1dB. First stage compression is achieved by biasing ZX60-V63+ to $1.8 \mathrm{~V}$, $-\mathrm{V} 82-\mathrm{S}+$ to $1.6 \mathrm{~V},-41-\mathrm{S}+$ to $1.5 \mathrm{~V}$, $-\mathrm{P} 33 \mathrm{ULN}+$ to $0.6 \mathrm{~V}$ and $-14012 \mathrm{~L}-\mathrm{S}+$ to $7 \mathrm{~V}$.

\begin{tabular}{|c|c|c|c|c|c|}
\hline & $\Delta_{f}=$ & 1 & 10 & 100 & $150[\mathrm{MHz}]$ \\
\hline ZX60- & & \multicolumn{4}{|c|}{ Output Compression $\Delta_{e}[\mathrm{~dB}]$} \\
\hline $\begin{array}{l}\text { (1) V82-S+ } \\
\text { (2) P33ULN+ }\end{array}$ & $\begin{array}{l}\text { (HBT) } \\
\text { (E-PHEMPT) }\end{array}$ & 1.7 & 2.1 & 3 & 1.1 \\
\hline $\begin{array}{l}\text { (1) } 43-\mathrm{S}+ \\
\text { (2) } 43-\mathrm{S}+\end{array}$ & $\begin{array}{l}\text { (HBT) } \\
\text { (HBT) }\end{array}$ & 3.1 & 2.7 & 3.8 & 6.4 \\
\hline $\begin{array}{l}\text { (1) } 14012 \mathrm{~L}-\mathrm{S}+ \\
\text { (2) P33ULN+ }\end{array}$ & $\begin{array}{l}(\text { HBT }) \\
\text { (E-PHEMPT) }\end{array}$ & 1.7 & 1.8 & 2.4 & 1.7 \\
\hline $\begin{array}{l}\text { (1) } 43-\mathrm{S}+ \\
\text { (2) P33ULN+ }\end{array}$ & $\begin{array}{l}(\text { HBT }) \\
\text { (E-PHEMPT) }\end{array}$ & 2 & 2 & 1.3 & 4.4 \\
\hline $\begin{array}{l}\text { (1) V63+ } \\
\text { (2) P33ULN+ }\end{array}$ & $\begin{array}{l}\text { (HBT) } \\
\text { (E-PHEMPT) }\end{array}$ & 2.3 & 2.4 & 1.5 & 4.4 \\
\hline $\begin{array}{l}\text { (1) } 14012 \mathrm{~L}-\mathrm{S}+ \\
\text { (2) } 43-\mathrm{S}+\end{array}$ & $\begin{array}{l}(\mathrm{HBT}) \\
(\mathrm{HBT})\end{array}$ & 2.6 & 2.0 & 4.0 & 5.7 \\
\hline $\begin{array}{l}\text { (1) V63+ } \\
\text { (2) V82-S+ }\end{array}$ & $\begin{array}{l}\text { (HBT) } \\
\text { (HBT) }\end{array}$ & 3.3 & 2.1 & 1.8 & 3.3 \\
\hline \multicolumn{2}{|l|}{ ZX60- } & \multicolumn{4}{|c|}{ Input Compression $\Delta_{e}[\mathrm{~dB}]$} \\
\hline $\begin{array}{l}\text { (1) V82-S+ } \\
\text { (2) P33ULN+ }\end{array}$ & $\begin{array}{l}\text { (HBT) } \\
\text { (E-PHEMPT) }\end{array}$ & 14.8 & 13.5 & 16.5 & 16.5 \\
\hline $\begin{array}{l}\text { (1) } 43-\mathrm{S}+ \\
\text { (2) } 43-\mathrm{S}+\end{array}$ & $\begin{array}{l}(\text { HBT }) \\
(\text { HBT })\end{array}$ & 14.4 & 14.2 & 20.1 & 16.6 \\
\hline $\begin{array}{l}\text { (1) } 14012 \mathrm{~L}-\mathrm{S}+ \\
\text { (2) P33ULN+ }\end{array}$ & $\begin{array}{l}\text { (HBT) } \\
\text { (E-PHEMPT) }\end{array}$ & 10.2 & 10.3 & 9.6 & 7.4 \\
\hline $\begin{array}{l}\text { (1) } 43-\mathrm{S}+ \\
\text { (2) P33ULN+ }\end{array}$ & $\begin{array}{l}\text { (HBT) } \\
\text { (E-PHEMPT) }\end{array}$ & 20.4 & 21.7 & 21.6 & 23.3 \\
\hline $\begin{array}{l}\text { (1) V63+ } \\
\text { (2) P33ULN+ }\end{array}$ & $\begin{array}{l}\text { (HBT) } \\
\text { (E-PHEMPT) }\end{array}$ & 16.2 & 15.6 & 18.9 & 18.8 \\
\hline $\begin{array}{l}\text { (1) } 14012 \mathrm{~L}-\mathrm{S}+ \\
\text { (2) } 43-\mathrm{S}+\end{array}$ & $\begin{array}{l}(\mathrm{HBT}) \\
(\mathrm{HBT})\end{array}$ & 10.1 & 8.31 & 0.6 & 2.3 \\
\hline $\begin{array}{l}\text { (1) V63+ } \\
\text { (2) V82-S+ }\end{array}$ & $\begin{array}{l}\text { (HBT) } \\
\text { (HBT) }\end{array}$ & 19.6 & 21.2 & 21 & 21.3 \\
\hline
\end{tabular}

[6] A. N. Atanasov, M. S. Oude Alink, and F. E. van Vliet, "Reverse intermodulation in multi-tone array transmitters," in 2020 IEEE BiCMOS and Compound Semiconductor Integrated Circuits and Technology Symposium (BCICTS), 2020, pp. 1-4.

[7] J. Martens and P. Kapetanic, "Probe-tone S-parameter measurements," IEEE Transactions on Microwave Theory and Techniques, vol. 50, no. 9, pp. 2076-2082, Sep. 2002.

[8] I. Angelov, L. Bengtsson, and M. Garcia, "Extensions of the chalmers nonlinear HEMT and MESFET model," IEEE Transactions on Microwave Theory and Techniques, vol. 44, no. 10, pp. 1664-1674, 1996.

[9] J. Verspecht, D. Barataud, J.-P. Teyssier, and J.-M. Nébus, "Hot S-parameter techniques: $6=4+2$," in 2005 66th ARFTG Microwave Measurement Conference (ARFTG), 2005, pp. 1-9.

[10] "ZX60-V63+," https://www.minicircuits.com/pdfs/ZX60-V63+.pdf.

[11] "ZX60-V82+," https://www.minicircuits.com/pdfs/ZX60-V82+.pdf.

[12] “ZX60-14012L-S+," https://www.minicircuits.com/pdfs/ ZX60-14012L-S+.pdf.

[13] “ZX60-43-S+,” https://www.minicircuits.com/pdfs/ZX60-43-S+.pdf.

[14] “ZX60-P33ULN+," https://www.minicircuits.com/pdfs/ZX60-P33ULN+ .pdf. 